\section{Uveitis and juvenile arthritis}

\section{James T Rosenbaum, Justine R Smith}

\section{The many guises of uveitis associated with JIA}

T he inflammatory joint disease currently referred to as juvenile idiopathic arthritis (JIA) has endured many labels including Still's disease, ${ }^{1}$ juvenile chronic arthropathy, and juvenile rheumatoid arthritis. ${ }^{3}$ From the work of Edelsten and colleagues published in this issue of the BJO (p 51), we learn that the ocular prognosis for this disease is as diverse as the names for the entity itself. Importantly, we also discover that certain clinical factors, most significantly, the severity of uveitis at onset, can help us predict prognosis.

Establishing a diagnosis is a means to an end that will guide both the physician and the patient in gauging prognosis and choosing among treatment options. But how is one to interpret a diagnosis that has a broad spectrum of outcomes? How does a clinician offer advice for a disease such as uveitis if one patient might suffer 48 hours of mild inflammation, followed by complete resolution, whereas another patient might endure 48 years of chronic inflammation and consequent blindness? Can research help recognise clues that will guide physician and patient in devising a treatment protocol such that "the punishment," otherwise known as the treatment, truly fits "the crime," also known as the disease.

The outcome of a disease is determined by many factors besides what is implied by the diagnosis itself. Both patients and physicians believe that treatment is a major determinant of outcome. The gold standard in assessing the effect of therapy is the randomised controlled clinical trial. In the case of uveitis, the clinician can rarely rely on this gold standard because it has seldom been undertaken. Since we believe that treatment is important, we naturally believe that compliance with treatment is important. Sex and other genetically determined factors, socioeconomic status, education, and the results of laboratory tests are additional factors that may influence or predict prognosis. The microarray gene chip technology that allows an investigator to determine expression of thousands of genes simultaneously promises to revolutionise the ability to determine prognosis. Gene array can be used to show that "one disease" based on clinical features and histopathology is more than one disease based on gene expression, as exemplified by diffuse large $B$ cell lymphoma. Furthermore, gene array promises to identify host genetic factors that may influence clinical outcome, just as the presence of sickle cell trait influences the course of malaria.

The report by Edelsten and colleagues is especially laudable for its approach. To derive its valuable conclusions, the authors needed a large database, recording careful observations over nearly two decades. A provocative conclusion is that the prognosis for the eye disease associated with JIA has improved over the past 17 years. Why should this be? It is tempting to congratulate ourselves that more aggressive treatment, such as with methotrexate, has been responsible. But one must remember that we do not have rigorous proof to demonstrate the efficacy of methotrexate for this or any other form of uveitis. It is tempting to hope that the new systemically administered inhibitors of tumour necrosis factor will improve prognosis even further, but early reports have been somewhat contradictory about the role for these drugs in the treatment of inflammatory eye disease. $^{5}$

\section{"The severity of uveitis ... can help us predict prognosis of juvenile idiopathic arthritis"}

Sometimes improved prognosis is a mirage induced by early detection. The prognosis for uveitis associated with JIA would mistakenly appear improved if more vigilant screening in 1998 allowed the ophthalmologist to diagnose the child with a mild cellular response in the anterior chamber that was destined to disappear regardless of treatment, while this same scenario in 1982 did not come to the attention of an ophthalmologist. Shifting diagnostic criteria may also explain an apparent change in disease prognosis over time. The term "juvenile idiopathic arthritis," was coined by the International League Against Rheumatism Task Force for Classification Criteria in 1995, when, recognising the inadequacy of previous terminology, it developed a system for classification of child- hood arthritis. ${ }^{6}$ This umbrella term acknowledges that different forms of arthritis are subsumed within it. Despite revision in 1997, ${ }^{7}$ the system continues to receive criticism and further revision is suggested. ${ }^{8}$ Further, it is clear that clinicians and scientists remain confused in the classification. For example, one might argue that in the study by Edelsten and co-workers, patients classified as "non-standard" because they had uveitis associated with psoriatic arthritis did, in fact, belong to the "standard" group, if psoriatic arthritis is considered a form of JIA as recommended by current classification criteria.

Edelsten and colleagues appropriately recognise the limitations of their study. Extrapolations to an individual practice must be made cautiously if the physician has a different threshold for initiating methotrexate, if follow up visits are scheduled less frequently, and if the definition of "severe disease" varies from that used in this report. Prognostic information is always based on probability. Patients are rarely interested to learn that the probability of blindness is $6 \%$, if the unfortunate individual in the examination chair today is that unlucky one person out of 17 .

With the publication of this report by Edelsten and co-workers, we know more about prognosis of JIA associated uveitis than we ever have before. We also know that we have much more to learn about this eye disease.

\section{ACKNOWLEDGMENTS}

JTR was supported by National Institute of Health (No EYO6484), Research to Prevent Blindness, and the Rosenfeld Family Fund.

Br J Ophthalmol 2002;86:1-2

\section{Authors' affiliations}

James T Rosenbaum, Justine R Smith, Casey Eye Institute, Oregon Health Sciences University, Portland, Oregon, USA

Correspondence to: Dr J T Rosenbaum, Casey Eye Institute, Oregon Health Sciences University, 3375 SW Terwilliger Blvd, Portland, OR 97201-4197, USA; rosenbaj@ohsu.edu

\section{REFERENCES}

1 Smiley WK. The eye changes in Still's disease. Proc Roy Soc Med 1957; 51 . 597-600.

2 Kanski JJ. Uveitis in juvenile chronic arthritis: incidence, clinical features and prognosis. Eye 1988;2:225-8

3 Nguyen QD, Foster CS. Saving the vision of children with juvenile rheumatoid arthritis-associated uveitis. JAMA 1998;280: 1 133-4.

4 Alizadeh AA, Eisen MB, Davis RE, et al. Distinct types of diffuse large B-cell lymphoma identified by gene expression profiling. Nature 2000;403:503-11.

5 Smith JR, Levinson RD, Holland GN, et al. Differential efficacy of tumor necrosis factor inhibition in the management of inflammatory eye disease and associated rheumatic disease. Arthritis Rheum 2001;45:252-7. 

6 Fink CW and the Task Force for Classification Criteria. Proposal for the development of classification criteria for idiopathic arthritides of childhood. J Rheumatol 1995;22:1566-9.

7 Petty RE and the Task Force for Classification Criteria. Revision for the proposed classification criteria for juvenile idiopathic arthritis: Durban, 1997. J Rheumatol 1998;25: 1991-4.
8 Fantini F. Classification of chronic arthritides of childhood (juvenile idiopathic arthritis): criticisms and suggestions to improve the efficacy of the Santiago-Durban criteria. J Rheumatol 2001;28:456-9.

\section{Outcome of corneal transplantation}

\section{Melissa M Brown, Gary C Brown}

\section{Value based ophthalmology}

n their article in this issue of the $B J O$ (p 57) Saunders and colleagues describe a methodology by which they evaluate the visual health state of patients with severe corneal disease requiring transplantation surgery. Rather than dealing with visual acuity as the only preoperative and outcome parameters, they evaluate patients according to three criteria: (1) visual acuity, (2) ocular pain, and (3) visual function. Concerning the latter criterion, visual function, they utilise a tool called the VFA (visual function assessment), ${ }^{1}$ which they have previously described; it is essentially a modification of the VF- $14^{2}$ and predominantly measures ocular function characterised by the ability to perform tasks such as driving, reading, cooking, etc. They found that patients who had a high preoperative priority score, as measured by the three above criteria, were more likely to have a good outcome. One measure in the study that is somewhat unclear, though, is how the results incorporate vision in the eye that did not receive a transplant. All too often our clinical trials and other studies fail to address the status of the second eye, perhaps a factor more important to the patient that the ocular intervention itself. The authors noted that $72.4 \%$ of patients demonstrated an improved VFA after transplant, but it is uncertain whether this was measured using only the operated eye or in a real world situation in which both eyes were used during the assessment.

The authors should be congratulated upon bringing more than visual acuity alone into the decision making process. Most ophthalmologists believe that the central visual acuity is the most important factor related to the quality of life of an ophthalmic patient. And over a century of experience suggests that they are probably right. But measurement of the visual acuity alone, while it typically is the primary benchmark for most evidence based data, does not necessarily provide the best value based data.

So the question arises, what is the difference between evidence based medicine and value based medicine, or in this case, evidence based ophthalmology and value based ophthalmology? Evidence based medicine incorporates the most reliable and reproducible data from clinical studies, particularly clinical trials. Value based medicine takes clinical efficacy delineated by evidence based data one step further and incorporates the evidence to measure the actual value of the therapy to a patient.

While the concept of value based medicine may sound nebulous, it is far from it. The concept of value can be quantified by assessing the improvement in length of life and/or quality of life conferred by an intervention. For there is really nothing else we do, or should do, in health care other than improve length and/or quality of life. With ophthalmological interventions, in which death is infrequently encountered, the value of an intervention can be essentially measured by the improvement in quality of life.

Why is value important? Every society has finite scarce resources that, in the best interest of its people, should be maximised to yield the highest return. This endeavour can be quite difficult in health care, in fact impossible unless value based health care is considered. As an example, suppose a financial officer responsible for a healthcare budget at a large company poses the following question to an ophthalmologist. "Doctor, you have just told me that laser treatment for macular oedema associated with branch retinal vein obstruction improves the average person's vision from 20/70 to $20 / 45$. What does this mean in terms of value? The cardiologists were in here just before you and said that their evidenced based therapy improves the cardiac ejection fraction from $35 \%$ to $45 \%$. What is the comparable value of your treatment and theirs?" With evidence based data alone, the question is virtually impossible to answer. With value based data it can be answered.

How does one measure value? Saunders and colleagues have attempted one method of measuring value by incorporating visual acuity, pain, and their visual function assessment as preoperative and postoperative criteria. The latter tool measures functional ability associated with various degrees of visual loss and has been considered by some to be a quality of life measure. We're not so sure, however, that it truly measures quality of life or value. We have previously noted a high correlation between the VF-14 and visual acuity, not surprising since those with better central visual acuity can perform more intricate visual tasks. The question can then be asked, how much more do such visual function tests really tell us than the central visual acuity alone? The answer is still uncertain. But perhaps more importantly, the visual function tests (VF-14, ${ }^{2}$ the VFQ- $25^{3}$ from the National Eye Institute and the visual function assessment ${ }^{1}$ ) most commonly used to evaluate quality of life for ophthalmological interventions are generally not applicable across other medical specialties. And as much as we like to think that health care revolves around ophthalmology, those involved with healthcare policy have a much broader picture with which to deal.

\section{"Data indicate that our interventions in ophthalmology are highly valued by patients"}

There is a tool, however, that can measure the value associated with a healthcare intervention. And it can measure it across virtually all interventions in medicine. This evaluation tool is utility analysis. ${ }^{4}$ Developed in the 1940s to measure uncertainty, utility analysis was applied by researchers to health care in the 1970s. By convention, a utility value of 1.0 equates with perfect health and a value of 0.0 equates with death. There are a number of variants of utility value measure; one of the more popular variants, the time tradeoff methodology, essentially involves a theoretical scenario 
in which a patient is asked how many expected remaining years of life he or she would be willing to trade in return for a perfect health state. The proportion of years traded is then subtracted from 1.0 to yield the utility value. For example, if a person with $20 / 40$ vision is willing to trade 4 of 20 hypothetical remaining years in return for perfect vision, the utility value would be $1.0-4 / 20=0.80$. Unlike many of the quality of life tools in health care that primarily measure function, utility analysis is theoretically more all inclusive in that it encompasses function, as well as other important parameters such as fear of the unknown, pain, psychological overlay, family support systems, socioeconomic status, and others. Of utmost importance is the fact that utility analysis can also be combined with the costs of an intervention in costutility analysis. ${ }^{5}$ Cost-utility analysis has the ability to assess the resources expended for the value received from an intervention and can effectively compare interventions across all healthcare fields.

Patient perceived value is a basic component in the evaluation of what we do in health care, perhaps one of the most critical. Data from ophthalmic populations to date indicate indeed that our interventions in ophthalmology ${ }^{45}$ are highly valued by patients. This information is of unsurpassed importance to stakeholders in the healthcare arena: patients, decision makers, planners, and providers as well. It should indeed be gratifying to those of us in the profession that the services we perform are so highly valued by the most important people in the process, our patients.

Many in health care refer to "evidence based medicine" as a very positive progression from the more anecdotal practice of medicine 20 years ago. But those in the business world and on the cutting edge of innovation speak of value. There is little doubt that, while "evidence based medicine" was the buzz phrase of the 1990s, moving forward it will be teamed with patient perceived value and cost-utility analysis to take healthcare quality to a yet higher level, "value based medicine" - the paradigm for the 21 st century.

Br J Ophthalmol 2002;86:2-3

\section{Authors' affiliations}

Melissa M Brown, Gary C Brown, Center for Evidence-Based Health Care Economics, Suite 210, 1107 Bethlehem Pike, Flourtown, PA 19031, USA

Correspondence to: Melissa Brown; Lissa1011@aol.com

\section{REFERENCES}

1 Courtright $\mathbf{P}$, Poon $\mathrm{Cl}$, Richards JSF, et al. Visual function among corneal disease patients waiting for penetrating keratoplasty in British Columbia. Ophthalmic Epidemiol 1998;5:13-20.

2 Broman AT, Munoz B, West SK, et al. Psychometric properties of the 25-item NEI-VFQ in a Hispanic population: Proyecto VER. Invest Ophthalmol Vis Sci 2001;42:606-13.

3 Steinberg EP, Tielsch JM, Schein OD, et al. The VF-14: an index of functional impairment in patients with cataract. Arch Ophthalmol 1994; 1 12:630-8.

4 Brown GC, Brown, MM, Sharma S. Health care in the 21 st century: evidence-based medicine, patient preference-based quality, and cost-effectiveness. Quality Management in Health Care 2000;9:23-31.

5 Busbee B, Brown MM, Brown GC, et al. Cost-effectiveness of initial cataract surgery. Ophthalmology (in press).

\section{Atherosclerotic cardiovascular disease and diabetic retinopathy}

\section{Jorge G Arroyo}

\section{Risk for the development and progression of retinopathy}

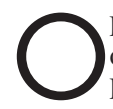

phthalmologists are well aware of the strong association between longstanding hyperglycaemia and the microvascular manifestations of diabetic retinopathy. ${ }^{2}$ The relation between macrovascular large vessel disease and diabetic retinopathy is less well understood, and is the subject of Klein $e t$ al's paper in this issue of the BJO (p 84). These authors seek to shed light on the association between arteriosclerotic cardiovascular disease and diabetic retinopathy in a cohort of patients from the Cardiovascular Health Study (CHS).

In their paper, Klein et al found an association between cardiovascular disease, elevated plasma LDL cholesterol and gross proteinuria, and diabetic retinopathy. These associations were independent of age, sex, race, blood sugar, and duration of diabetes. They did not, as one might expect, find an association between internal carotid artery wall abnormalities or subclinical atherosclerosis and diabetic retinopathy. Although there was a significant association between elevated systolic blood pressure and retinopathy in the univariate analysis, this relation lost significance in the multivariate analysis. In contrast, elevated blood pressure has been found to be significantly associated with diabetic retinopathy in various large prospective and cross sectional studies. ${ }^{34}$ Klein et al found a significant relation between elevated plasma LDL cholesterol and retinopathy, which is consistent with other studies. ${ }^{56}$ This finding will be more definitively answered in ongoing prospective randomised controlled clinical trials of lipid lowering drugs. Finally, the association between gross proteinuria and diabetic retinopathy found by Klein et al corroborates the findings of other investigators. $^{78}$

As the authors point out, there are some limitations to their study. Firstly, and probably most significantly, is the fact that only about one half of the patients with diabetes, 296 out of 558 people classified as having diabetes in the CHS, were evaluated. The diabetic participants excluded from the study were in general older and sicker than the participants who were studied. Therefore, the younger and healthier diabetic participants in the study were not representative of the entire cohort of diabetic people in the CHS. Secondly, the cohort of diabetic participants studied is relatively small. Finally, the nonmydriatic non-stereoscopic fundus photograph of one eye of each participant probably underreported the prevalence of diabetic retinopathy and macular oedema in the participants.

More definitive demonstration of the relation of atherosclerotic cardiovascular disease and diabetic retinopathy will require long term prospective studies begun at or before the onset of diabetes. Until these studies are actualised, Klein et al's findings provide us with tantalising evidence of an association between atherosclerosis and diabetic retinopathy. If their results are confirmed by future studies, diabetic patients with evidence of atherosclerosis may have a higher risk for the development and progression of 
retinopathy and may require more frequent examinations and interventions.

Br J Ophthalmol 2002;86:3-4

\section{Author's affiliations}

Jorge G Arroyo, Department of

Ophthalmology, Massachusetts Eye and Ear

Infirmary, Brigham and Women's Hospital, and

Beth Israel Deaconess Medical Center, Harvard

Medical School, Boston, MA, USA

Correspondence to: Retina Service, 12th Floor, Massachusetts Eye and Ear Infirmary, Boston, MA 02114 , USA;

jarroyo@caregroup.harvard.edu

\section{REFERENCES}

1 DCCT Research Group. The effect of treatment of diabetes on the development and progression of long-term complications in insulin-dependent diabetes mellitus. N Engl J Med 1993:329:977-86

2 UKPDS Group. Intensive blood-glucose control with sulphonylureas or insulin compared with conventional treatment and risk of complications in patient with type 2 diabetes UKPDS 33. Lancet 1998;352: 837-53.

3 UKPDS Group. Tight blood pressure control and risk macrovascular and microvascular complications in type 2 diabetes. UKPDS 38 BM 1998:317:703-13

4 UKPDS Group. Diabetic retinopathy at diagnosis of non-insulin dependent diabetes mellitus and associated risk factors. UKPDS 30. Arch Ophthalmol 1998;116 297-303.

5 ETDRS Group. Association of elevated serum lipid levels with retinal hard exudate in diabetic retinopathy. ETDRS 22 Arch Ophthalmol 1996;114: 1079-84.

6 WESDR Group. Relationship of serum cholesterol to retinopathy and hard exudate. Ophthalmology 1991;98:1261-5.

7 WESDR Group. Is gross proteinuria a risk factor for the incidence of proliferative diabetic retinopathy. Ophthalmology 1993; 100:1140-6

8 Jensen T, Deckert T. Diabetic retinopathy, nephropathy and neuropathy. Horm Metab Res Suppl 1992;26:68-70.

\section{7th European Forum on Quality Improvement in Health Care}

We are delighted to announce this forthcoming conference to be held in Edinburgh, Scotland on 21-23 March 2002. Delegate enquiries are welcome.

The themes of the Forum are:

- Leadership, culture change and change management

- Achieving radical improvement by redesigning care

- Health policy for lasting improvement in health care systems

- Patient safety

- Measurement for improvement, learning and accountability

- Partnership with patients

- Professional quality: the foundation for improvement

- Continuous improvement in education and training

- People and improvement

Presented to you by the BM Publishing Group (London, UK) and Institute for Healthcare Improvement (Boston, USA).

For more information contact: quality @bma.org.uk or look at the website www.quality. bmipg.com. Tel: +44 (0)20 7383 6409; fax: +44 (0)20 73736869 